\title{
Simulação Numérica de Trocador de Calor Solo-Ar Constituído por \\ Formas Geométricas Complexas
}

\section{Numerical simulation of Earth-Air Heat Exchanger Constituted by Compound Geometric Shapes}

Michel Kepes Rodrigues¹, Bruna Rodrigues Nunes², Luiz Alberto Oliveira Rocha ${ }^{3}$, Elizaldo Domingues dos Santos ${ }^{4}$, Liércio André Isoldi 5

\section{RESUMO}

O Trocador de Calor Solo-Ar (TCSA) é aplicado para promover uma melhoria da condição térmica no interior de edificações. O objetivo deste estudo é avaliar a influência da forma geométrica da instalação do TCSA em seu desempenho através de parâmetros construtivos e operacionais. Para isso, 5 instalações de TCSA são analisadas empregando a simulação numérica e métodos analíticos. Os resultados mostram que a presente técnica permite uma análise multiobjetivos, levando em consideração as restrições físicas e geométricas do sistema e fornecendo uma contribuição prática, ao levar em consideração aspectos de design e operacionais do TCSA, e uma contribuição teórica na modelagem de turbulência.

Palavras-chave: Simulação numérica, formas geométricas, parâmetros construtivos.

\section{ABSTRACT}

The Earth-Air Heat Exchanger (EAHE) is applied to promote a thermal condition improvement inside buildings. The objective of this study is to evaluate the influence of geometric shape of EAHE installation in its performance by means constructive and operational parameters. To do so, 5 EAHE installations are analyzed employing numerical simulation and analytical methods. The results show that the present technique allows a multiobjective analysis, taking into account the physical and geometric constraints of the system and providing practical contribution in taking into account design and operational aspects of EAHE and a theoretical contribution in turbulence modeling.

Keywords: Numerical simulation, geometric shapes, constructive parameters.
${ }^{1}$ Doutorando, Programa de PósGraduacão em Modelagem Computacional da Universidade Federal do Rio Grande (FURG)

E-mail:

michel.professor@yahoo.com.br

2 Mestre, Programa de PósGraduação em Modelagem Computacional da Universidade Federal do Rio Grande (FURG)

3 Doutor, Programa de PósGraduação em Engenharia Mecânica da Universidade do Vale do Rio dos Sinos (UNISINOS)

4 Doutor, Programa de PósGraduação em Modelagem Computacional da Universidade Federal do Rio Grande (FURG)

5 Doutor, Programa de PósGraduação em Modelagem Computacional da Universidade Federal do Rio Grande (FURG) 


\section{INTRODUÇAOO}

O Trocador de Calor Solo-Ar (TCSA) é um dispositivo constituído por um ou mais dutos enterrados no solo, através dos quais o ar flui movido pela ação de ventiladores. Esse dispositivo é capaz de aproveitar a energia térmica armazenada na camada superficial do solo, proveniente da incidência da radiação solar e, com isso, reduzir o consumo de energia elétrica dos equipamentos de condicionamento de ar tradicionalmente utilizados para a climatização de edificações. O princípio de funcionamento do TCSA baseia-se na transferência de calor do solo para o ar que escoa nos dutos, em períodos frios, e do escoamento de ar para o solo, em períodos quentes, de acordo com as estações do ano. Com isso, a temperatura do ar que sai do TCSA é sempre mais amena em relação à temperatura do ar ambiente que entra no TCSA (Rodrigues et al., 2015; Rodrigues et al., 2015a).

O objetivo deste trabalho é estudar a influência de diferentes geometrias de instalação de um TCSA, com a finalidade de reduzir o volume ( $V$ ) de solo necessário para o sistema de TCSA, reduzir a perda de carga $(P C)$ do ar ao fluir dentro do duto e aumentar o potencial térmico $(P T)$ do TCSA. Por se tratar de uma análise multiobjetivo, busca-se a melhor adequação entre esses parâmetros. Para isso, são propostas 5 instalações de TCSA com formas geométricas distintas, sendo a com geometria mais simples considerada como referência. Para promover este estudo, são empregados métodos analíticos, para a determinação da perda de carga e do volume ocupado, e numérico, para a obtenção do potencial térmico. Além disso, dados experimentais são adotados para a validação e como condições de contorno de temperatura prescrita do modelo computacional.

O trabalho proposto justifica-se, uma vez que, na literatura, são encontrados vários trabalhos abordando casos de disposição geométrica (horizontal, vertical) e configurações geométricas (retangulares, triangulares, em série, paralelas) de TCSA; entretanto, não são encontrados trabalhos com instalações de TCSA com um elevado nível de complexidade na configuração geométrica, onde parâmetros construtivos e operacionais sejam simultaneamente considerados. Esse estudo, então, traz um tema original de pesquisa, que acredita-se ser relevante para o Estado da Arte de TCSA, já que nenhuma referência abrangendo este tópico foi encontrada na literatura (Sobti e Singh, 2015; Gupta e Tiwari, 2016). 


\section{MATERIAIS E METODOS}

A Figura 1 mostra o domínio computacional e as condições de contorno do modelo empregado neste trabalho, que foi desenvolvido no software FLUENT, para a simulação numérica do princípio de funcionamento de um TCSA.

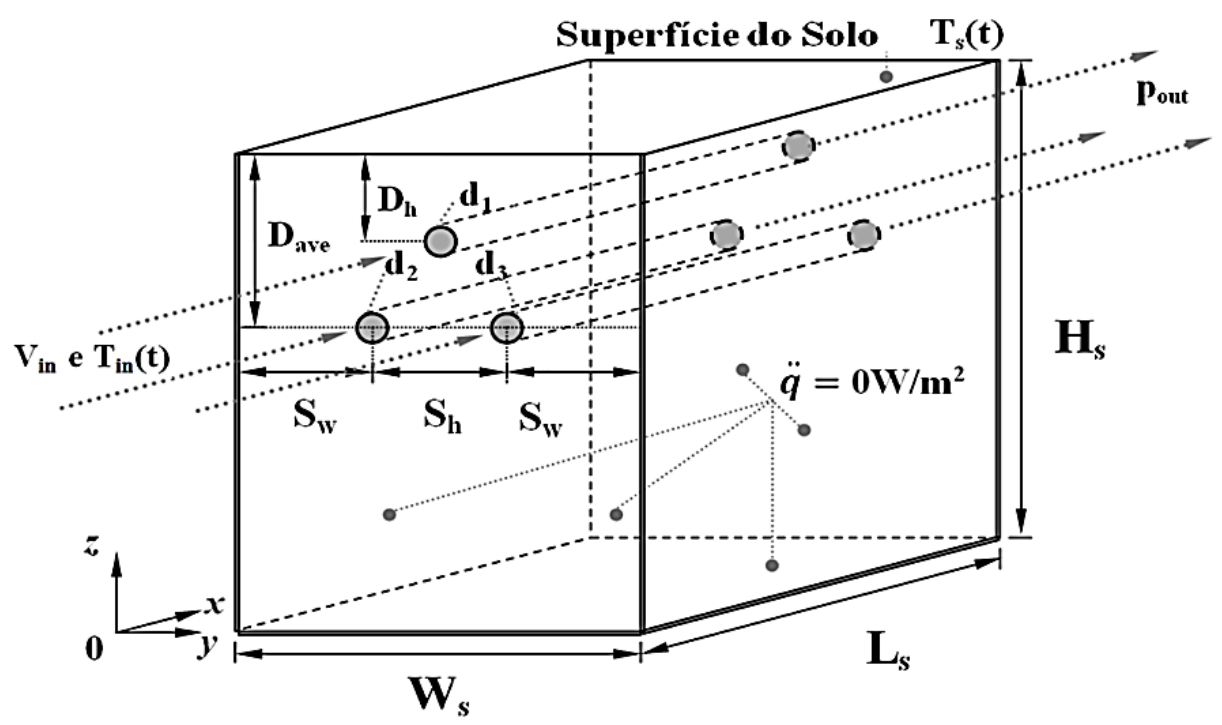

Figura 1. Domínio computacional do TCSA.

Como pode ser visto, o duto $\left(d_{1}\right)$ é enterrado na profundidade relativa $\left(D_{h}\right)$ e os dutos $\left(d_{2}\right.$ e $\left.d_{3}\right)$ são enterrados na profundidade média (ou de referência) $\left(D_{\text {ave }}\right.$ ) igual a 3,00 m em relação à superfície do solo, já que não há ganho significativo no $P T$ do TCSA para maiores valores de profundidade (Brum et al., 2012). O comprimento total do duto é representado por $(L)$. O espaçamento horizontal entre a parede do duto e a parede do domínio computacional $\left(S_{w}\right)$ é sempre igual a 2,00 m, sendo esse o valor mínimo para evitar a influência da condição de contorno de fluxo prescrito nulo nos resultados numéricos (Rodrigues et al. 2015b). Já para o espaçamento horizontal $\left(S_{h}\right)$ entre dois dutos em paralelo, adota-se como $1,00 \mathrm{~m}$ a distância mínima para que não ocorra influência significativa na troca de calor entre os dutos, que resultaria em uma redução do PT do TCSA (Marzarotto et al., 2015). Portanto, neste estudo, o valor de $S_{h}$ igual a 1,00 m é utilizado na construção das geometrias do TCSA.

Neste estudo, o comprimento $\left(L_{s}\right)$, a largura $\left(W_{s}\right)$ e a profundidade $\left(H_{s}\right)$ definem 0 volume ( $V$ do solo ocupado pela instalação de TCSA (ver Fig. 1). Como não há variação significativa na temperatura do solo para profundidades superiores a $H_{s}=15,00 \mathrm{~m}$ (Brum et al. 2012; Brum et al., 2013), esse valor foi utilizado em todas as instalações estudadas. 
Já os valores de $L_{s}$ e $W_{s}$ de cada instalação são definidos de acordo com sua forma geométrica, respeitando os valores mínimos de $S_{w}$ e $S_{h}$ citados anteriormente.

Para as instalações propostas neste estudo, a velocidade de entrada de ar ( $\left.V_{\text {in }}\right)$ no duto é de $3,3 \mathrm{~m} / \mathrm{s}$, a pressão do ar de saída do duto (pout) é a pressão atmosférica, as temperaturas do ar na entrada do duto e na superfície do solo são prescritas e representadas por funções periódicas ajustadas estatisticamente (pelo Método dos Mínimos Quadrados) e inseridas no modelo computacional através de funções definidas pelo usuário (UDF - User Defined Functions), com base nos dados experimentais de Vaz et al. (2011) e Vaz et al. (2014). A condição de fluxo de calor prescrito nulo foi imposta nas paredes laterais e na superfície inferior do volume do solo do domínio computacional (Rodrigues et al., 2015; Rodrigues et al., 2015a).

$\mathrm{Na}$ abordagem computacional não foi considerada a espessura da parede do duto (de PVC) no modelo, admitindo-se que o ar escoa por meio de perfurações cilíndricas inseridas diretamente no volume do solo e em contato direto com o solo. Essa simplificação já foi adotada em trabalhos anteriores (Vaz et al., 2011; Isoldi et al., 2013; Brum et al., 2013; Rodrigues et al., 2015; Rodrigues et al., 2015a) e não altera significativamente a solução final do problema, viabilizando a geração da malha no domínio computacional. As propriedades termofísicas do solo e do ar são mostradas na Tabela 1.

Tabela 1. Propriedades termofísicas dos materiais.

\begin{tabular}{c|c|c|c|c}
\hline Materiais & $\begin{array}{c}\text { Massa } \\
\text { específica } \\
\rho\left(\mathrm{kg} / \mathrm{m}^{3}\right)\end{array}$ & $\begin{array}{c}\text { Condutividade } \\
\text { térmica } \\
k(\mathrm{~W} / \mathrm{m} \cdot \mathrm{K})\end{array}$ & $\begin{array}{c}\text { Calor específico a } \\
\text { pressão constante } \\
c_{p}(\mathrm{~J} / \mathrm{kg} \cdot \mathrm{K})\end{array}$ & $\begin{array}{c}\text { Viscosidade } \\
\text { dinâmica } \\
\mu \mathrm{kg} /(\mathrm{m} \cdot \mathrm{s})\end{array}$ \\
\hline Solo & 1.800 & 2,1 & 1.780 & $-\cdot-\cdot-\cdot-\cdot---$ \\
\hline $\mathrm{Ar}$ & 1,160 & 0,0242 & 1.010 & $1,798 \times 10^{-5}$ \\
\hline
\end{tabular}

A perda de carga $(P C)$ do ar ao escoar no interior dos dutos no TCSA é determinada seguindo a metodologia analítica tradicional dos escoamentos internos. Em escoamentos internos, turbulentos, totalmente desenvolvidos, a $P C$ pode ser avaliada de forma distribuída (trechos retos) e localizada (curvas, conexões, etc.). Portanto, a contabilização de todas as perdas associadas a componentes localizados, mais as perdas distribuídas da tubulação definem o valor total da $P C$. Para o cálculo da $P C$ distribuída, foram considerados os seguintes valores: diâmetro $d=110 \mathrm{~mm}$, rugosidade $\varepsilon=0,420$ $\mathrm{mm}$, número de Reynolds $R e=23.531,91$, definindo um fator de atrito $f=0,027$. Já para a 
determinação da perda de carga localizada, coeficientes de perda de carga localizada $k=$ 0,3 e $k=0,2$ para curvas com 90 e com 180ㅜ, respectivamente, foram considerados. Maiores informações sobre como determinar o fator de atrito estão em: Bergman et al. (2011) e Pritchard e Mitchell (2015).

Cabe destacar que a $P C$ no TCSA é um parâmetro operacional importante que permite a investigação da variação da pressão do ar dentro do duto e a determinação/seleção da potência do ventilador necessária para que o ar possa superar a resistência interna ao escoamento. Sabiamente, à medida que a PC no TCSA aumenta, a energia elétrica consumida pelo ventilador também é aumentada gradativamente.

O PT, por sua vez, é um indicador para avaliar o desempenho térmico do TCSA e pode ser determinado a partir de uma diferença média de temperaturas. $O P T$ da instalação de TCSA, formada por um único duto, pode ser expresso como a média quadrática (RMS - Root Meam Square) da diferença entre a temperatura do ar na saída (calculada numericamente) e na entrada (condição de temperatura prescrita) do TCSA, ao longo do ano. Portanto, em períodos quentes do ano, tem-se o PT de resfriamento; nos períodos frios do ano, tem-se o PT de aquecimento; e em períodos intermediários pode haver a condição de equilíbrio térmico em que o $P T=0$. Cabe destacar que nas simulações numéricas foram obtidos resultados de temperaturas a cada $21.600 \mathrm{~s}$, totalizando 1.460 medições (Rodrigues et al., 2015a). Para simular o comportamento térmico e, consequentemente, obter os valores de temperatura do ar na saída do TCSA ao longo do tempo, a ferramenta utilizada neste estudo é a modelagem computacional (Patankar, 1980; Versteeg e Malalasekera, 2007).

\subsection{Modelagem matemática}

Para obtenção do campo de temperaturas no solo, é utilizada a equação da conservação da energia (Bergman et al., 2011; Versteeg e Malalasekera, 2007). Para tratar do escoamento do ar no interior do duto na condição de regime turbulento, escoamento incompressível, transiente, com transferência de calor, são aplicadas as equações de conservação da massa, da quantidade de movimento e da energia (Wilcox, 2002; Versteeg e Malalasekera, 2007; Bergman et al., 2011; Pritchard e Mitchell, 2015). Para abordar a turbulência é aplicado o modelo $k$ - $\varepsilon$ padrão (Wilcox, 2002; Launder e Salding, 1972). 


\subsection{Modelagem computacional}

No pré-processamento é empregado o software GAMBIT, o qual possibilita a construção das geometrias, a definição dos tipos de condições de contorno e a geração das malhas computacionais. E o software FLUENT promove a realização do processamento e do pós-processamento. O software FLUENT baseia-se no método dos volumes finitos (FVM - Finite Volume Method). No pós-processamento também é utilizado o software EXCEL. O FVM é utilizado neste trabalho já que atende, em nível de volumes elementares, os princípios de conservação da massa, da quantidade movimento e da energia e é uma ferramenta importante no campo da dinâmica dos fluidos computacional (CFD - Computational Fluid Dynamics). Para a realização das simulações numéricas é adotado um passo de tempo de 3.600 s (uma hora) em um total de 17.520 passos de tempo. A temperatura de inicialização do modelo computacional é de $18,7^{\circ} \mathrm{C}$ (temperatura média no solo). As soluções numéricas são consideradas convergidas quando os resíduos para a equação de conservação da massa e da quantidade de movimento são inferiores a $10^{-3}$ e para a equação de conservação da energia são inferiores a $10^{-6}$ entre duas iterações consecutivas. Mais informações sobre a abordagem numérica podem ser encontradas em Rodrigues et al. (2015) e Rodrigues et al. (2015a).

A técnica de refinamento sucessivo é adotada para obter a malha computacional independente. Isso é feito aumentando o número de células computacionais da malha atual para a próxima malha até que uma diferença relativa, para a temperatura do ar $(T)$ de saída do TCSA, entre dois refinamentos consecutivos de malha seja de no máximo 0,2\%. A Tabela 2 traz o teste de independência de malha e a definição do critério para estabelecer o processo de criação de malha com volumes tetraédricos, que é $d / 3$ e $3 d$ para o duto e o solo, respectivamente, onde d é o diâmetro interno do duto. Esse refinamento de malha computacional já foi adotado em trabalhos anteriores sobre TCSA (Isoldi et al., 2013; Vaz et al., 2011; Rodrigues et al., 2015; Rodrigues et al. 2015a) e é empregado nas simulações numéricas realizadas neste estudo. 
Tabela 2. Teste de independência de malha.

\begin{tabular}{|c|c|c|c|}
\hline \multicolumn{2}{|c|}{$\begin{array}{l}\text { Refinamento de malha } \\
(\mathrm{mm})\end{array}$} & \multirow{2}{*}{$\begin{array}{c}\text { Temperatura de } \\
\text { saída do TCSA } \\
\left({ }^{\circ} \mathrm{C}\right)\end{array}$} & \multirow{2}{*}{$\begin{array}{c}\text { Diferença relativa (\%) } \\
\qquad\left|\frac{T_{j}-T_{j+1}}{T_{j}}\right| 100\end{array}$} \\
\hline Duto & Solo & & \\
\hline$d / 3$ & $3 d$ & 25,40 & 0,197 \\
\hline $4 d / 17$ & $12 d / 17$ & 25,45 & 0,039 \\
\hline $5 d / 26$ & $15 d / 26$ & 25,46 & - \\
\hline
\end{tabular}

Os dados experimentais de Vaz et al. (2011) e Vaz et al. (2014) são utilizados para validar o modelo computacional empregado neste trabalho com base em Rodrigues et al. (2015). Para tanto, uma instalação simplificada (ver Fig. 1), em relação à complexa apresentada por Vaz et al. (2011) e Vaz et al. (2014), foi utilizada.

Por brevidade, será apresentado aqui somente o processo referente à análise de validação e verificação do modelo computacional para o duto $\left(d_{3}\right)$, sendo que as análises para os dutos $d_{1}$ e $d_{2}$ são omitidas. O processo de verificação e validação completo está apresentado em Rodrigues et al. (2015).

Assim, adotou-se $L_{s}=25,77 \mathrm{~m}, W_{s}=10,00 \mathrm{~m}, H_{s}=15,00 \mathrm{~m}, L=25,77 \mathrm{~m}, d_{3}=110$ $\mathrm{mm}, v_{\text {in }}=3,30 \mathrm{~m} D_{\text {ave }}=1,60 \mathrm{~m}, S_{h}=0,60 \mathrm{~m}, D_{h}=0,50 \mathrm{~m}$ (ver Fig. 1). A Figura 2 mostra a variação da temperatura de saída de ar do duto $\left(d_{3}\right)$ do TCSA em função do tempo, permitindo a comparação entre dados experimentais de Vaz et al. (2011) e Vaz et al. (2014), e os resultados numéricos deste trabalho. Cabe destacar que uma curva foi ajustada, estatisticamente, aos dados experimentais através do Método dos Mínimos Quadrados (LSM - Least Squares Method). Além disso, é apresentada também a solução numérica referente ao trabalho de Rodrigues et al. (2015).

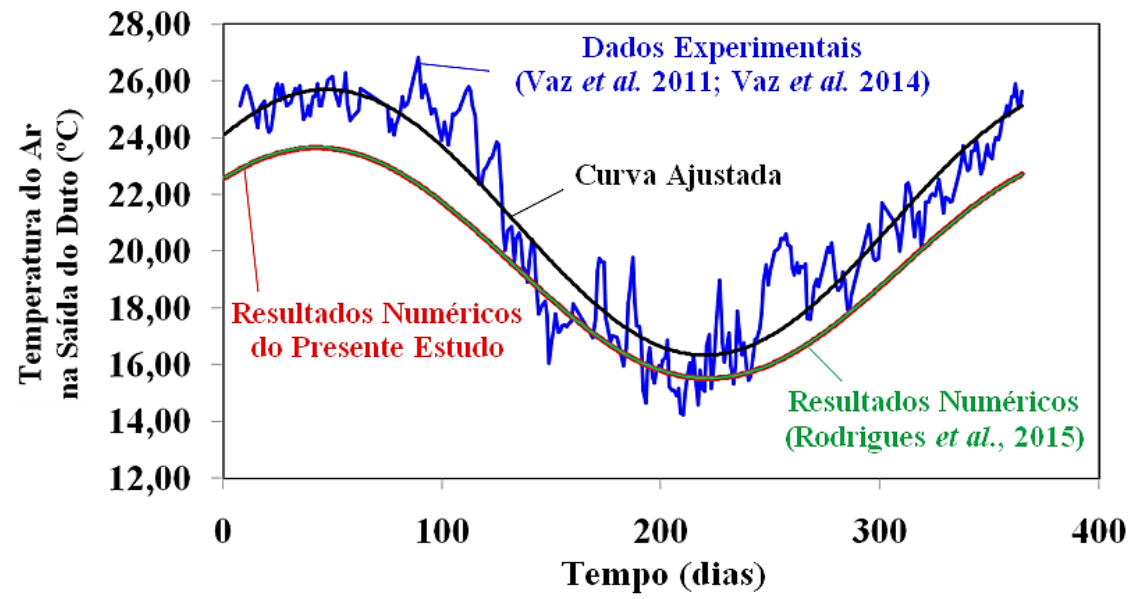

Figura 2. Processo de validação e verificação do modelo numérico de TCSA. 
Comparando, na Fig. 2, os resultados numéricos obtidos no presente estudo com os de Rodrigues et al. (2015) observa-se uma excelente concordância entre eles (coeficiente de correlação de Pearson de 0,998). É importante destacar ainda que o tempo de processamento do modelo computacional do presente trabalho, que adota o modelo $k-\varepsilon$, é reduzido em aproximadamente $20 \%$ em relação ao modelo computacional de Rodrigues et al. (2015) que utiliza o modelo de tensões de Reynolds (RSM - Reynolds Stress Model). Além disso, simulações numéricas de instalações de TCSA com configuração geométrica mais complexas não convergem quando modelo de turbulência RSM é empregado.

Já quando os resultados obtidos no presente estudo são comparados aos dados experimentais de Vaz et al. (2011) e Vaz et al. (2014) uma diferença média absoluta de $7,2 \%$ (em torno de $1,60^{\circ} \mathrm{C}$ ) é observada e um coeficiente de correlação de Pearson de 0,933 é encontrado em relação à curva ajustada aos dados experimentais. Portanto, o modelo computacional de TCSA empregado neste trabalho, com base em Brum et al. (2012) e Brum et al. (2013), pode ser considerado verificado e validado.

\section{INSTALAÇÖES DE TROCADOR DE CALOR SOLO-AR ESTUDADAS}

Cinco diferentes formas geométricas para o TCSA foram consideradas. A mais simples, mostrada na Fig. 3, é um TCSA com duto reto, e será usada como referência, denominada, portanto, de Instalação Referência (IR).

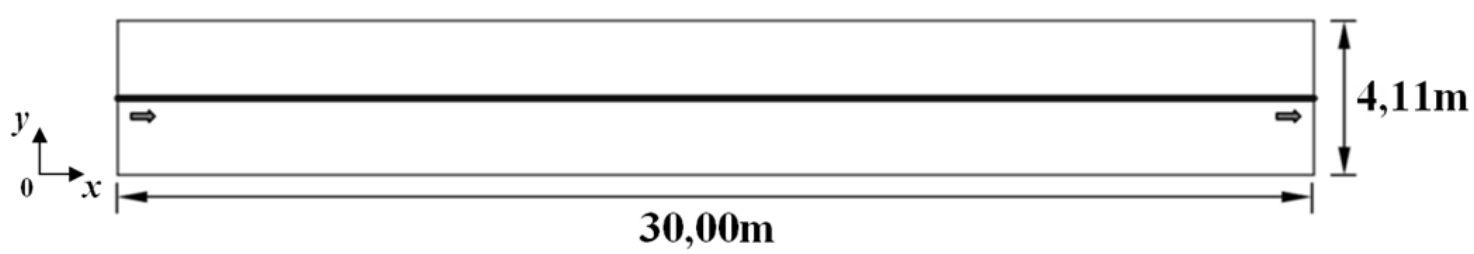

Figura 3. Vista superior da Instalação Referência (IR).

E, na Fig. 4 as outras 4 formas geométricas para o TCSA são mostradas. 


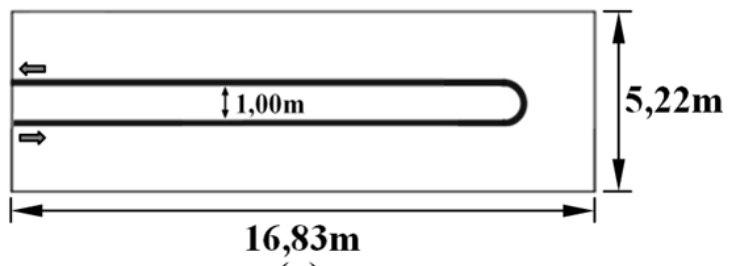

(a)
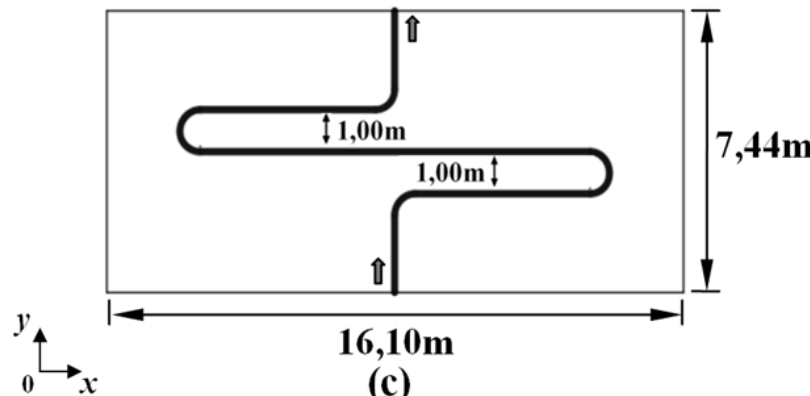

(c)

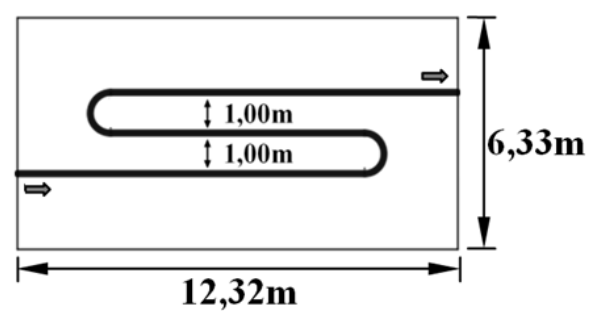

(b)

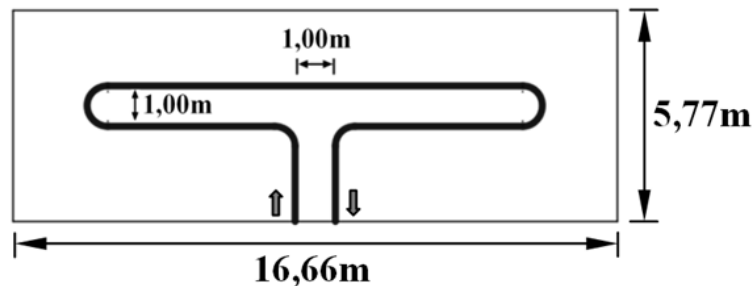

(d)

Figura 4. Vista superior das formas geométricas para o TCSA: (a) Instalação 1 (11), (b) Instalação 2 (I2), (c) Instalação 3 (I3) e (d) Instalação 4 (14).

Cabe destacar que em todas as instalações de TCSA propostas nesse trabalho (ver Figs. 1, 3 e 4) mantém os seguintes parâmetros fixos: $H_{s}=15,00 \mathrm{~m}, d=110,00 \mathrm{~mm}$, $V_{\text {in }}=3,30 \mathrm{~m}$ e $D_{\text {ave }}=3,00 \mathrm{~m}$. Além disso, todas as instalações possuem um comprimento total de duto de $30,00 \mathrm{~m}$. Já os valores de $L_{s}$ e $W_{s}$ (Figs. 3 e 4 ) foram determinados a partir da configuração geométrica de cada instalação, respeitando as restrições impostas de $S_{w}=2,00 \mathrm{~m}, S_{h}=1,00 \mathrm{~m}$.

\section{RESULTADOS E DISCUSSÄO}

Os resultados são baseados na análise dos seguintes parâmetros: o volume de solo $(V)$, a perda de carga $(P C)$ e o potencial térmico $(P T)$ de cada instalação de TCSA proposta. A fim de possibilitar uma comparação global entre as instalações estudadas, esses parâmetros são normalizados em relação à IR (mostrada na Fig. 3).

Então, na Figura 5 (a) é possível observar que a 13 apresenta a maior ocupação no volume de solo, enquanto a 12 oferece a menor ocupação no volume de solo em relação às IC. Entretanto, a IR proporciona a maior ocupação no volume de solo em relação às instalações estudadas. Portanto, a 12 consegue oferecer uma redução no volume de solo ocupado de $36,76 \%$ em relação à $I R$ e de $34,90 \%$ em relação à 13 . 


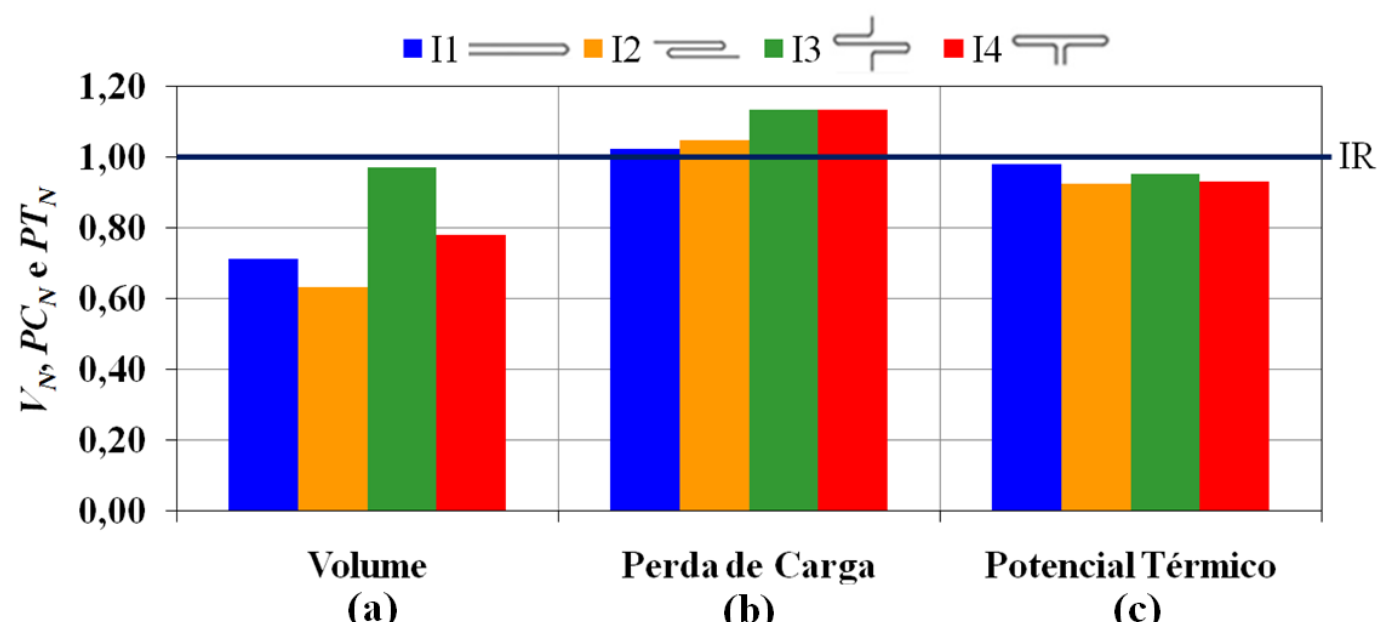

(a)

(b)

(c)

Figura 5. Condição de volume de solo ocupado (a), de perda de carga (b) e potencial térmico (c) das instalações de TCSA.

A Figura 5 (b) mostra que as 13 e 14 causam a maior perda de carga, enquanto a I1 proporciona a menor perda de carga. No entanto, a IR, devido à simplicidade na forma geométrica, apresenta menor perda de carga em relação às instalações analisadas. Dessa forma, a I1 apresenta um aumento na perda de carga de 2,44\% em relação à IR e uma redução de $9,68 \%$ em ralação às 13 e 14 . Nessa análise a presença da maior quantidade no número curvas das 13 e 14 , em relação às demais instalações estudadas, impõem aumento significativo na perda de carga do TCSA.

E na Figura 5 (c) nota-se que o menor potencial térmico observado se dá na I2, enquanto o maior é obtido na I1. Contudo, a IR proporciona o maior PT do TCSA em relação às instalações estudadas. Assim, a 11 oferece uma redução de apenas 1,85\% em relação ao $P T$ do TCSA da IR e a 12 apresenta uma redução de 5,66\% em relação ao $P T$ do TCSA da 12. A troca de calor, portanto, entre os trechos paralelos das IC de TCSA, embora não influenciando de forma significativa no $P T$ do TCSA, implicam em uma pequena queda de desempenho térmico devido à diminuição do volume de solo utilizado pelo TCSA.

Por fim, para definir entre os casos estudados qual deles possui o melhor desempenho global, considerando o problema multiobjetivo, foi realizada uma análise vetorial. Busca-se aqui a instalação que conduza à minimização de $V N$, minimização de $P C N$ e maximização de $P T N$ (ou minimização de 1/PTM) simultaneamente.

A partir dos resultados da Tabela 2, a instalação que mais se aproxima da instalação considerada ideal (que minimizaria totalmente $V N, P C N$ e 1/PTN, gerando um 
vetor nulo) é a 11, pois possui o menor valor na análise vetorial realizada. Portanto, é possível afirmar que a 11 possui o melhor desempenho global entre as configurações geométricas propostas na Fig. 4.

Tabela 2. Definição da instalação de TCSA com melhor desempenho global.

\begin{tabular}{c|c|c|c|c}
\hline Instalação & $V_{N}$ & $P C_{N}$ & $1 / P T_{N}$ & Vetor \\
\hline I1 & 0,71 & 1,02 & 1,02 & 1,61 \\
\hline I2 & 0,63 & 1,05 & 1,08 & 1,63 \\
\hline I3 & 0,97 & 1,13 & 1,05 & 1,82 \\
\hline I4 & 0,78 & 1,13 & 1,07 & 1,75 \\
\hline
\end{tabular}

Assim, na Fig. 6 é apresentado o efeito térmico proveniente da troca de calor na I1 para um dia típico de verão. A partir da entrada do ar no duto, se dá uma progressiva e contínua dissipação térmica do ar para o solo, na direção do eixo $x$, e uma redução da intensidade do campo de temperaturas, na direção do eixo $y$, no sentido do escoamento do ar no interior do duto, ao visualizar no plano longitudinal $x y$, em $D_{a v e}=3,00 \mathrm{~m}$. Nesse caso específico, o ar entra no TCSA com uma temperatura em torno de $30^{\circ} \mathrm{C}$ e sai com temperatura em torno de $21^{\circ} \mathrm{C}$. Essa diferença de $9^{\circ} \mathrm{C}$ permite uma redução, significativa, no consumo de energia elétrica em um equipamento de condicionamento de ar que tradicionalmente é empregado na climatização de edificações.

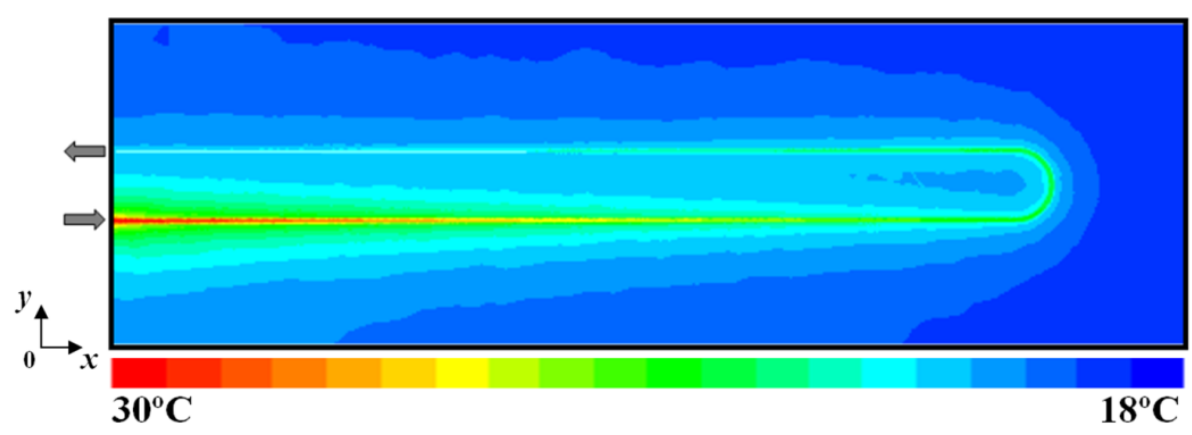

Figura 6. Condição do potencial térmico para a I1 de TCSA.

\section{CONSIDERAÇOES FINAIS}

Os resultados obtidos nesse trabalho mostram que se não houver restrição em relação ao volume de solo ocupado pelo TCSA, a IR é a melhor opção. Com ela, a perda de carga é mínima e o potencial térmico é máximo. 
Entretanto, considerando a utilização do TCSA e zonas urbanas, onde os terrenos possuem dimensões limitadas, torna-se necessário adotar configurações geométricas de maior complexidade que um simples duto reto (usado na IR).

Nesse sentido, avaliando separadamente no TCSA a influência do volume de solo ocupado, da perda de carga imposta ao escoamento e do potencial térmico, recomendase a utilização das instalações I2, I1, e I1, respectivamente. Entretanto, se uma análise multiobjetivo é realizada, indica-se o emprego da 11 .

Portanto, conclui-se que é fundamental avaliar a influência dos parâmetros operacionais e construtivos do TCSA, de maneira a obter uma performance superior do mesmo.

Além disso, o presente estudo também traz uma contribuição teórica na modelagem computacional de sistemas TCSA com configuração geométrica complexa, indicando a utilização modelo de turbulência $k-\varepsilon$. Esse modelo permite simular computacionalmente o comportamento térmico e fluidodinâmico do ar em diferentes instalações de TCSA. Também se observa uma redução de aproximadamente $20 \%$ no tempo de processamento de simulação numérica ao utilizar o modelo de turbulência k- $\varepsilon$, em vez do modelo de turbulência RSM.

Pretende-se, em trabalhos futuros, dar continuidade a esse trabalho empregando a metodologia apresentada aqui associada ao método Design Construtal (Bejan e Lorente, 2008), visando a otimização geométrica de TCSA.

\section{AGRADECIMENTOS}

Os autores agradecem ao Conselho Nacional de Desenvolvimento Científico e Tecnológico (CNPq) pelo apoio financeiro através do Projeto Universal (processo: 445558/2014-8) e à Fundação de Amparo à Pesquisa do Estado do Rio Grande do Sul (FAPERGS) pelo apoio financeiro através do Edital Pesquisador Gaúcho (termo de outorga: 17/2551-0001). E. D. dos Santos, L. A. Isoldi e L. A. O. Rocha agradecem ao CNPq por suas bolsas de Produtividade em Pesquisa. 


\section{REFERENCIAS}

BEJAN, A.; LORENTE, S. Design with Constructal Theory. Hoboken: Wiley, 2008.

BERGMAN, T. L.; LAVINE, A. S.; INCROPERA, F. P.; DEWITT, D. P. Fundamentals of Heat and Mass Transfer. John Wiley \& Sons Inc., Hoboken, $7^{\text {a }}$ Ed., p. 1048, 2011.

BRUM, R. S.; ROCHA, L. A. O.; VAZ, J.; DOS SANTOS, E. D.; ISOLDI, L. A. Development of Simplified Numerical Model for Evaluation of the Influence of Soil-Air Heat Exchanger Installation Depth over its Thermal Potential. International Journal of Advanced Renewable Energy Research, n. 1, p. 505-514, 2012.

BRUM, R. S.; VAZ, J.; ROCHA, L. A. O.; DOS SANTOS, E. D.; ISOLDI, L. A. A New Computational Modeling to Predict the Behavior of Earth-Air Heat Exchangers. Energy and Buildings, n. 64, p. 395-402, 2013.

GUPTA, N.; TIWARI, G. N. Review of Passive Heating/Cooling Systems of Buildings. Energy Science \& Engineering, Society of Chemical Industry, John Wiley \& Sons Ltd, n. 29, 2016.

ISOLDI, L. A.; BRUM R. S.; RODRIGUES M. K.; RAMALHO J. V. A.; VAZ, J.; SOUZA, J. A.; DOS SANTOS, E. D.; ROCHA, L. A. O. Constructal Design of Earth-Air Heat Exchangers. In: Constructal Law Conference. Proceedings of Constructal Law Conference, Nanjing, n. 1, p. 88-96, 2013.

LAUNDER, B. E.; SALDING, D. B. Lectures in Mathematical Models of Turbulence. London: Academic Press, 1972.

MARZAROTTO, C.; NUNES, B.; RODRIGUES, M. K.; SOUZA, J. A.; ROCHA, L. A. O.; BRUM, R. S.; DOS SANTOS, E. D.; ISOLDI, L. A. Numerical Analysis of the Influence of Operational and Constructive Parameters on the Operation of Earth-Air Heat Exchangers. Scientia Plena, v. 11, n. 10, 2015.

PATANKAR, S. Numerical Heat Transfer and Fluid Flow. New York, USA: McGraw-hill, 1980.

PRITCHARD, P. J.; MITCHELL, J. W. Fox and Mcdonald's: Introduction To Fluid Mechanics. 9ª Ed., John Wiley \& Sons Inc., Hoboken, 2015.

RODRIGUES, M. K.; BRUM, R. S.; VAZ, J.; ROCHA, L. A. O.; DOS SANTOS, E. D.; ISOLDI, L. A. Numerical Investigation About the Improvement of the Thermal Potential of an Earth-Air Heat Exchanger (EAHE) Employing the Constructal Design Method. Renewable Energy, n. 80, p. 538-551, 2015.

RODRIGUES, M. K.; DOS SANTOS, E. D.; ISOLDI, L. A. Numerical Analysis and Constructal Design of Earth-Air Heat Exchanger: Mathematical Methods for Engineering, $1^{\underline{a}}$ ed. Sarbrücken: Lap Lambert Academic Publishing, v. 1, p. 167, $2015 a$.

RODRIGUES, M. K.; SOUZA, J. A.; VAZ, J.; JUNIOR, I. C. A.; ROCHA, L. A. O.; BRUM, R. S; DOS SANTOS, E. D.; ISOLDI, L. A. Numerical Study of the Influence of Boundary Conditions on the Computational Modeling of Earth-Air Heat Exchanger. Scientia Plena, $11,10,2015 b$. 
SOBTI, J.; SINGH, S. K. Earth-Air Heat Exchanger as a Green Retrofit for Chandīgarh - A Critical Review. Geohermal. Energy, n. 9, 2015.

VAZ, J.; SATTLER, M. A.; DOS SANTOS, E. D.; ISOLDI, L. A. Experimental and Numerical Analysis of an Earth-Air Heat Exchanger. Energy and Buildings, n. 43, p. 2476-2482, 2011.

VAZ, J.; SATTLER, M. A.; BRUM, R. S.; DOS SANTOS, E. D.; ISOLDI, L., A. An experimental study on the use of earth-Air heat exchangers (EAHE). Energy and Buildings, n. 72, p.122-131, 2014.

VERSTEEG, H.; MALALASEKERA, W. An Introduction to Computational Fluid Dynamics: The Finite Volume Method. England: Pearson Education Limited, 2007.

WILCOX, D. C. Turbulence Modeling For CFD. California, EUA: DCW Industries, 2002. 